\title{
A RECUPERAÇÃO DE SERVIÇOS COMO FERRAMENTA DE RELACIONAMENTO E SEU IMPACTO NA CONFIANÇA E LEALDADE DOS CLIENTES
}

\section{RESUMO}

O objetivo central deste trabalho é examinar a recuperação de serviços sob uma perspectiva estratégica, como ferramenta de relacionamento. Para tanto, foi proposto e testado um modelo teórico, com foco nos inter-relacionamentos entre avaliações específicas do processo de reclamação, confiança, custo de mudança, valor e lealdade do consumidor. Os resultados indicam que a formação de confiança e lealdade do consumidor é afetada pela forma como as reclamações são resolvidas. As percepções de justiça afetaram a satisfação com o gerenciamento da reclamação. A confiança do consumidor foi fortemente influenciada pela satisfação pós-reclamação. Por fim, tanto a intenção de recompra como a comunicação boca-a-boca foram influenciadas por confiança, satisfação e valor percebido. Os custos de mudança não moderaram as relações entre satisfação, confiança e lealdade, e demonstraram ter baixa influência nas intenções de recompra, indicando que a criação de mecanismos que dificultem a saída do cliente não é suficiente para mantê-lo, e que e um gerenciamento adequado da reclamação pode ser uma ferramenta eficaz para desenvolver relacionamentos de longo prazo.

\section{Cristiane Pizzutti dos Santos \\ UFRGS \\ Daniel Von der Heyde Fernandes \\ UFRGS}

\begin{abstract}
The main goal of this paper is to examine service recovery under a strategic perspective, as a relationship tool. A theoretical model has been proposed and tested, with focus on interrelationships among specific evaluations of complaint process, trust, switching costs, perceived value, and loyalty. The findings indicate that customer trust and loyalty are affected by the way complaints are resolved. Perceptions of justice affected satisfaction with complaint management. Consumer trust is strongly influenced by post-complaint satisfaction. Finally, both intentions of repurchase and word-of-mouth communication were influenced by trust, satisfaction and perceived value. Switching costs did not moderate the relationships between satisfaction, trust and loyalty, and demonstrated to have low influence on intentions of repurchase. Therefore, the creation of mechanisms that inhibit customers' exit is not enough keep them, and an adequate complaint management may be an efficient tool to develop long-termed relationships.
\end{abstract}

PALAVRAS-CHAVE Recuperação de serviços, lealdade, confiança, satisfação, relacionamento com clientes. KEYWORDS Service recovery, loyalty, trust, satisfaction, customer relationship. 


\section{INTRODUÇÃO}

Atualmente, observa-se crescente ênfase, tanto no meio acadêmico como no empresarial, em estratégias ligadas ao marketing de relacionamento (BREI; ROSSI, 2005). Tais estratégias, em vez de focarem a obtenção de novos clientes no mercado, ou a atração de clientes de outras empresas, buscam reduzir o êxodo dos consumidores atuais, evitando que mudem de marca, e estimulando sua fidelidade e seu desejo de manter transações com a empresa.

No contexto brasileiro, há uma profusão de artigos sobre marketing de relacionamento (p. ex., SLONGO; LIBERALI, 2004; BREI; ROSSI, 2005) que indicam o interesse pelo tema. As empresas percebem crescentemente que uma variedade de negócios depende dos clientes atuais, e satisfeitos. Segundo Tschohl (1991), cerca de 65\% dos negócios de uma empresa são freqüentes. Nesse ambiente de negócios em que se destaca a manutenção de clientes, cresce a importância dos episódios de reclamações. Diante deles, a empresa tem o potencial de restaurar a satisfação do consumidor, evitar a propaganda boca-aboca negativa, reforçar a lealdade e minimizar uma situação insatisfatória que poderia motivar o cliente a transferir suas transações para uma empresa concorrente.

A literatura sobre insatisfação e reclamação tem passado por importantes desenvolvimentos conceituais (STEPHENS; GWINNER, 1998; SINGH; WILKES, 1996) e empíricos (TAX; BROWN; CHANDRASHEKARAN, 1998; SMITH; BOLTON; WAGNER, 1999; MAXHAM; NETEMEYER, 2002; RINGBERG; ODEKERKEN; CHRISTENSEN, 2007). O papel tático da recuperação de serviços é claro: em um evento de falha de serviço, as empresas devem se comprometer a prover o serviço de maneira adequada em uma segunda tentativa (BROWN; COWLES; TUTEN, 1996). Ademais, sob a perspectiva estratégica, a recuperação de serviços foca o relacionamento com o cliente, em que o objetivo da recuperação não é somente corrigir uma falha específica, mas também aperfeiçoar o sistema de entrega de serviços (MAXHAM; NETEMEYER, 2002). O objetivo é diminuir as ocorrências de falhas ao longo do tempo, fazendo com que a percepção do consumidor em relação à qualidade do serviço se torne mais positiva e fortalecendo relacionamentos a longo prazo.

O intuito do presente trabalho é contribuir para o conhecimento sobre recuperação de serviços sob a perspectiva estratégica por meio do exame da recuperação de serviços como ferramenta de relacionamento. Sob tal prisma, é relevante investigar qual a influência dos esforços de recuperação de serviços sobre a lealdade do cliente, em comparação ao impacto exercido por outros fatores - confiança, valor percebido e custos de mudança. A proposta é analisar, empiricamente, uma estrutura teórica em que se considerem os inter-relacionamentos entre avaliações específicas do processo de reclamação, confiança, custo de mudança, valor e lealdade do consumidor. Tal estrutura é investigada em dois ambientes de serviços: companhias aéreas e bancos, com o objetivo de aumentar a abrangência do fenômeno investigado.

\section{FUNDAMENTAÇÃO TEÓRICA E HIPÓTESES DE PESQUISA}

A seguir, são abordados os principais conceitos tratados neste artigo: recuperação de serviços, percepção de justiça, satisfação após a falha, confiança e lealdade do consumidor, e as hipóteses elaboradas a partir da base teórica apresentada.

\section{Recuperação de serviços}

A recuperação de serviços refere-se às ações tomadas por uma empresa em resposta a uma falha no serviço (TAX et al., 1998). O processo de recuperação pode ser iniciado pelo próprio provedor do serviço ou pelo cliente. Quando se tem amplo contato com o cliente, como, por exemplo, no setor das companhias aéreas, em que um problema como atraso num vôo é aparente para ambas as partes, a recuperação é geralmente iniciada pelo provedor de serviços. Já em outros setores, é provável que a falha só seja conhecida pelo provedor de serviços quando o cliente solicitar uma ação corretiva, como é o caso de uma reclamação referente a um conserto feito em um automóvel. Alguns autores (BROWN et al., 1996; SMITH et al., 1999) diferenciam essas duas maneiras de se começar um processo de recuperação, considerando a primeira - ação que a empresa inicia no tempo e no lugar em que uma falha de serviço ocorreu - como "recuperação de serviço" (service recovery) e a segunda - ação em reação a uma reclamação sobre uma falha de serviço -, como "gerenciamento de reclamação" (complaint management). No entanto, com freqüência têm-se usado essas expressões como sinônimas (MAXHAM; NETEMEYER, 2002; CHEBAT; SLUSARCZYK, 2005; RINGBERG et al., 2007), como se faz neste artigo.

No cenário nacional, o reconhecimento dos direitos básicos do consumidor por meio do Código de Defesa do Consumidor, aprovado no início dos anos 1990, e o surgimento e a atuação de órgãos governamentais de defesa do consumidor, como os Procons, fazem com que os 
consumidores se tornem mais exigentes e conscientes de seus direitos. As empresas, por sua vez, procuram se ajustar a essa demanda da sociedade e do mercado por meio da criação de departamentos e serviços de atendimento ao consumidor (DAC ou SAC) e da disponibilidade de centrais telefônicas específicas para a resolução de problemas (SANTOS; ROSSI, 2002).

\section{Justiça e satisfação com 0 gerenciamento de reclamações}

Criada a partir dos estudos sobre transação social, a teoria da justiça tem contribuído na investigação da resolução de conflitos. Estudos contemporâneos sobre gerenciamento de reclamações oferecem evidências da adequação do conceito de justiça como base para entender o processo de recuperação de serviços e seus resultados (GOODWIN; ROSS, 1992; BLODGETT et al., 1997; TAX et al., 1998; SMITH et al., 1999). Tal conceito envolve três diferentes dimensões. Observando a ordem cronológica em que aparecem na literatura, a primeira dimensão a ser explorada foi a justiça distributiva, enfatizada pela teoria da eqüidade. Justiça distributiva refere-se à alocação de benefícios e custos entre as partes de uma transação (TAX et al., 1998). No contexto da reclamação, as distribuições são vistas como os resultados tangíveis oferecidos pela empresa ao reclamante, como, por exemplo, a troca do produto ou o dinheiro de volta (TAX et al., 1998).

A segunda dimensão é a justiça processual. Essa dimensão diz respeito às políticas e procedimentos usados pelas empresas durante os processos de reclamações e engloba seis subdimensões: flexibilidade, acessibilidade, controle de processo, controle de decisão, velocidade de resposta e aceitação de responsabilidade (THIBAUT; WALKER, 1975; BLOGGETT et al., 1997; TAX et al., 1998).

A justiça interpessoal é a terceira dimensão de justiça e envolve a maneira como os empregados tratam e se comunicam com os consumidores durante o episódio de reclamação. Seis subdimensões têm sido estudadas: cortesia, honestidade, empatia, esforço, oferecimento de explicações e pedido de desculpas (CLEMMER, 1988; TAX et al., 1998).

A satisfação pós-compra (ou inicial) tem sido considerada a mediadora principal entre crenças anteriores à compra e estruturas cognitivas pós-compra, comunicações e comportamento de recompra (WESTBROOK, 1987). Similarmente, a satisfação com o gerenciamento da reclamação (ou satisfação final) pode ser considerada um elemento central que media a relação entre avaliações sobre esse gerenciamento e atitudes e comportamentos pós-reclamação.

De acordo com a literatura sobre a justiça social, a satisfação está relacionada a avaliações de justiça em várias situações de conflitos (MESSICK; COOK, 1983). Estendendo essa lógica ao gerenciamento de reclamações, reconhece-se que a satisfação do consumidor com um episódio da reclamação é conseqüente à avaliação de aspectos que envolvem: o resultado final; o processo que levou a tal resultado; e a maneira como a empresa tratou o consumidor; e com ele se comunicou durante o episódio. Especificamente, a satisfação decorre da avaliação do quanto esses aspectos foram justos (GOODWIN; ROSS, 1992; BLODGETT et al., 1997; TAX et al., 1998; SMITH et al., 1999). Dessa forma se assume que cada dimensão da justiça - distributiva, processual e interpessoal - influencia a satisfação dos consumidores em relação à resolução de suas reclamações.

A magnitude do efeito de cada uma dessas dimensões na satisfação tem sido fator de divergência entre os pesquisadores da área. Tax et al. (1998) e Blogdett et al. (1997) descobriram que o efeito da justiça interpessoal na satisfação com o gerenciamento das reclamações é maior em magnitude do que o efeito das justiças distributiva e processual. Nos estudos de Goodwin e Ross (1992), Smith et al. (1999), e Santos e Rossi (2002), a justiça distributiva explicou uma parte relativamente maior do efeito total da justiça percebida na satisfação. Em função desses resultados contraditórios, não há conclusões prévias sobre a comparação entre os efeitos das três dimensões de justiça na satisfação, sendo apenas possível apresentar o primeiro grupo de hipóteses deste trabalho, a seguir:

$\mathrm{H}_{1}$ : Percepções de justiça interpessoal exercem impacto positivo na satisfação com o gerenciamento da reclamação.

$\mathrm{H}_{2}$ : Percepções de justiça processual exercem impacto positivo na satisfação com o gerenciamento da reclamação.

$\mathrm{H}_{3}$ : Percepções de justiça distributiva exercem impacto positivo na satisfação com o gerenciamento da reclamação.

\section{Satisfação com o gerenciamento da reclamação e confiança do consumidor}

A confiança do consumidor pode ser definida como "as expectativas, mantidas pelo consumidor, de que o provedor de serviço é responsável e pode ser confiável na entrega de suas promessas" (SIRDESHMUKH et al., 2002, p. 17). 
Estudos conceituais (NOOTEBOOM et al., 1997) e empíricos (MORGAN; HUNT, 1994; TAX et al., 1998; GARBARINO; JONHSON, 1999; AGUSTIN; SINGH, 2005; CHIOU; DROGE, 2006) têm destacado a confiança como elemento fundamental no desenvolvimento de fortes e longos relacionamentos entre consumidores e organizações. Berry (1995, p. 242) afirma que "a inerente natureza dos serviços, juntamente com abundante desconfiança na América, coloca a confiança como talvez a ferramenta mais poderosa de marketing de relacionamento disponível para uma empresa".

Ressalte-se que a confiança do consumidor no contexto de serviços tem sido estudada como um conceito bidimensional, em que uma dimensão diz respeito à confiança nos funcionários da linha de frente e a outra se relaciona às políticas da empresa prestadora de serviços. A inclusão de duas dimensões nas avaliações dos consumidores sobre serviços é defendida por autores como Doney e Cannon, 1997; e Sirdeshmukh et al., 2002. Estes últimos validaram essa distinção no contexto de serviços mediante as diferenças encontradas nos efeitos da confiança na empresa e da confiança nos empregados quanto à lealdade.

O papel da satisfação como elemento central na mediação entre as percepções dos consumidores sobre a recuperação de serviços e as atitudes e comportamentos futuros tem sido amplamente validado (BITNER et al., 1990; TAX et al., 1998; WEBSTER; SUNDARAM, 1998; DUBE; MAUTE, 1998). No entanto, o foco tem sido maior nas intenções imediatas de comportamento frente ao produto ou serviço em questão - como intenções de recompra, por exemplo - do que em variáveis que revelem o potencial para relacionamentos a longo prazo, como a confiança (RINGBERG et al., 2007). O trabalho desenvolvido por Tax et al. (1998) é um dos poucos que exploram a relação entre satisfação e variáveis relacionais. Eles encontraram uma significativa e positiva relação entre satisfação e confiança pós-reclamação, destacando o papel central da satisfação com o gerenciamento de conflitos na promoção (ou redução) da confiança entre as partes envolvidas.

Isso pode ser explicado pelo fato de que, quando o consumidor percebe a performance da empresa como justa e satisfatória, seus sentimentos de confiança tendem a ser reforçados. Situações de conflito podem ser ainda mais relevantes nesse sentido, sob a idéia de que é na adversidade que os parceiros são postos à prova (SMITH et al., 1999). Dessa forma, resolver satisfatoriamente uma falha na entrega de um produto, por exemplo, pode impactar mais a credibilidade de determinada empresa do que uma situação de rotina. Com base nessa lógica, sugere-se que:
$\mathrm{H}_{4}$ : A satisfação do consumidor com o gerenciamento da reclamação exerce impacto positivo em sua confiança.

\section{Lealdade e seus antecedentes: satisfação, confiança, valor e custos de mudança}

Neste trabalho, a lealdade do consumidor é definida como uma intenção comportamental de manter um relacionamento duradouro com o fornecedor de serviços (SIRDESHMUKH et al., 2002). A lealdade apresenta diversas conseqüências comportamentais e atitudinais ao consumidor. Entre elas estão a recompra, menor sensibilidade a preço, formação de laços afetivos e recomendações da empresa por meio de comunicação boca-a-boca positiva (SIRDESHMUKH et al., 2002; Oliver, 1999; DWYER; SCHURR; OH, 1987). Especial atenção tem sido dedicada à intenção de recompra e à comunicação boca-a-boca, apontadas como dimensões da lealdade em estudos recentes (LAM et al., 2004; SIDERSHMUKH et al., 2002; ZEITHAML et al., 1996). Seguindo essa mesma perspectiva, essas duas dimensões são utilizadas para medir a lealdade nesta pesquisa.

A partir dos resultados de outros estudos (WEBSTER; SUNDARAM, 1998; DUBE; MAUTE, 1998), propõe-se que a satisfação pós-reclamação influencia positivamente a lealdade. A lógica desse relacionamento se baseia na teoria de transações sociais, que sugere que ambas as partes envolvidas em uma troca estão motivadas a retribuir o tratamento e os benefícios obtidos nessa troca (THIBAUT; KELLY, 1959). Dessa forma, os reclamantes procuram retribuir os esforços da empresa em restaurar sua satisfação por meio da repetição da compra ou de uma positiva comunicação boca-a-boca.

Há uma profusão de pesquisas sobre as conseqüências das avaliações de satisfação no contexto de reclamações. Os resultados têm demonstrado que a satisfação com o gerenciamento da reclamação influencia positivamente nas futuras intenções de recompra e recompra real dos reclamantes (GILLY; GELB, 1982; MARTIN; SMART, 1994; BLODGETT et al., 1997); e também na comunicação boca-a-boca (TAX e CHANDRASHEKARAN, 1992; BLODGETT et al., 1997; SPRENG et al., 1995).

Espera-se que o construto confiança do consumidor também influencie positivamente a lealdade. A confiança oferece uma garantia quanto ao desempenho consistente e competente da empresa, garantindo que o consumidor continue a obter valor em negócios futuros com o mesmo fornecedor (SIRDESHMUKH et al., 2002). Reduzindo o risco nas trocas, a confiança contribui para dar continuidade à relação e criar sentimentos de lealdade. Assim, quanto maior a confiança do consumidor na empresa, 
maior a probabilidade de que realize futuros negócios com ela e mantenha um relacionamento de longo prazo. Os consumidores também tendem a comentar e recomendar a amigos e parentes as empresas nas quais têm grande confiança.

Assim, propõe-se que:

$\mathrm{H}_{5}$ : A satisfação do consumidor com o gerenciamento da reclamação influencia positivamente (a) sua intenção de recompra e (b) a comunicação boca-a-boca.

$\mathrm{H}_{6}$ : A confiança do consumidor influencia positivamente (a) a intenção de recompra e (b) a comunicação boca-a-boca.

Além da influência da confiança e da satisfação final na lealdade do consumidor, este estudo propõe a existência de um terceiro precursor da lealdade do consumidor: o valor percebido. Valor percebido refere-se à avaliação do consumidor quanto aos benefícios e custos de se manter um relacionamento com a empresa (SIRDESHMUKH et al., 2002). A lógica aqui é de que, mesmo quando o consumidor extrai benefícios de trocas relacionais com uma empresa, se lhe forem impostos altos custos de manutenção, o relacionamento será menos atrativo, e menores serão as evidências de lealdade - aqui entendidas como recompra e comunicação boca-a-boca. Os resultados obtidos por SIRDESHMUKH et al. (2002) oferecem evidência empírica desse raciocínio. Neste trabalho, o valor relacional emerge como um significante antecedente da lealdade do consumidor, independentemente da categoria de serviço. Com base nesse raciocínio, apresenta-se a seguinte hipótese:

$\mathrm{H}_{7}: \mathrm{O}$ valor percebido influencia positivamente (a) a intenção de recompra e (b) a comunicação de boca-a-boca.

Outro fator que desempenha papel importante na retenção de clientes, mas não necessariamente na comunicação boca-a-boca, são os custos de mudança. Segundo Fornell (1992, p. 10), os custos de mudança "fazem com que seja custoso para o consumidor trocar de fornecedor". Eles podem ser utilizados pelas empresas como ferramentas dentro de uma defensiva estratégia de marketing para manter clientes, forçando os concorrentes a investir na conquista desses clientes, menos dispostos a mudar de fornecedor do que consumidores que não enfrentam custos de mudança (FORNELL, 1992).

Segundo Burnham et al. (2003, p. 110), "à medida que os consumidores percebem custos de mudança ou barreiras para deixar um fornecedor, ficam inclinados a permanecer com o fornecedor". Pesquisas recentes têm confirmado essa relação, mostrando que os custos de mudança têm um efeito significativo na retenção dos clientes. Podem-se citar os resultados de Burnham et al. (2003) - em que os custos de mudança tiveram impacto maior na intenção de permanecer com o fornecedor do que a própria satisfação - e de Lam et al. (2004), que encontraram um efeito dos custos de mudança tão grande quanto o da satisfação na lealdade. Caruana (2003) encontrou uma relação positiva entre lealdade e custos de mudança. Logo, espera-se que:

$\mathrm{H}_{8}$ : Quanto maiores os custos de mudança, maior a intenção de recompra do cliente.

Também se encontra na literatura um efeito de interação entre custos de mudança e satisfação na retenção de clientes. Especificamente, em situações em que há altos custos de mudança, outros fatores tendem a desempenhar um papel menos importante na retenção do cliente do que nas situações com custos de mudança pequenos ou inexistentes. Tal interação foi encontrada por Jones et al. (2000), mas não foi confirmada por Burnham et al. (2003).

Não há, na literatura consultada, investigação que relacione custos de mudança, satisfação com o gerenciamento da reclamação e lealdade. Contudo, alguns estudos (ver FORNELL, 1992; JONES et al., 2000; LEE; LEE; FEICK, 2001) mostram que a satisfação com o produto ou serviço, assim como outros fatores atitudinais, torna-se menos importante na presença de custos de mudança. Portanto, propõe-se que:

$\mathrm{H}_{9}$ : Quanto maiores os custos de mudança, menor a influência da satisfação com o gerenciamento da reclamação na intenção de recompra.

$\mathrm{H}_{10}$ : Quanto maiores os custos de mudança, menor a in-

fluência da confiança na intenção de recompra.

Baseando-se na fundamentação teórica e nas hipóteses estabelecidas, a Figura 1 apresenta a estrutura teórica a ser investigada.

\section{MÉTODO DE PESQUISA}

Realizou-se um estudo descritivo de corte transversal com clientes de empresas aéreas e de bancos que se engajaram em processos de reclamação em relação a serviços prestados por empresas desses setores nos últimos 12 meses em relação à data da pesquisa. 
Esses serviços foram selecionados com base na tipologia de serviços de Bowen (1990), um dos poucos esquemas de classificação de serviços abrangentes e com bases empíricas. Essa classificação se baseia primariamente nos graus de customização e de contato presentes no contexto do serviço a ser prestado. Escolheram-se bancos e companhias aéreas para representar dois dos três tipos de serviços encontrados por Bowen. Os bancos são serviços pouco customizados e têm contato entre baixo e moderado com os clientes; enquanto as companhias aéreas prestam serviços padronizados e possuem contato de moderado a alto com os clientes. Além disso, acredita-se que esses dois contextos de serviços apresentam custos de mudança para os clientes, tais como programas de fidelidade e benefícios exclusivos no caso das companhias aéreas; e relacionamento com gerente e taxas de encerramento e abertura de conta, no caso dos bancos. As comparações entre os resultados referentes às companhias aéreas e aos bancos aumentam a possibilidade de generalização ou abrangência do modelo teórico testado.

O ambiente de negócio dos bancos é caracterizado pela alta concorrência, resultado da ampla entrada de bancos estrangeiros no mercado, pelo grande número de aquisições e fusões, e pelos bons resultados alcançados por significativa parcela das grandes empresas do setor no ano passado (ABARCA; ALDUNATE, 2004). Uma pesquisa de dezembro de 2005 do Procon de São Paulo revelou que, naquele ano, os bancos foram alvo de $12 \%$ das reclamações registradas, evidenciando um alto volume de queixas sobre o setor. Ainda segundo Abarca e Aldunate (2004), apenas $29 \%$ dos consumidores consideram que há um forte compromisso dos bancos em solucionar problemas.

Sobre o setor de companhias aéreas, deve-se destacar que, após o incidente de 11 de setembro, a aviação civil foi mundialmente abalada; e as conseqüências negativas sobre o setor ainda são sentidas (FELDMAN, 2002). Além disso, no Brasil, a presença de empresas novas - como Gol, a BRA e a Web Jet -, a crise na Varig e a desregulamentação da área têm instigado a competição entre as empresas (ARIENTE et al., 2005). Tal quadro indica elevada competitividade nos dois setores, o que implica a necessidade de as empresas estabelecerem relacionamento com os clientes e aumenta a relevância do gerenciamento de reclamações.

Levando-se em consideração o escasso número de estudos sobre a recuperação de serviços no Brasil (SANTOS; ROSSI, 2002), realizou-se uma fase exploratória de 12 entrevistas em profundidade com reclamantes dos setores em questão, no intuito de se levantarem questões relevantes sobre o processo de reclamação que envolve os serviços prestados por empresas aéreas e bancos. A fase exploratória proporcionou o refinamento das dimensões a serem incluídas na fase quantitativa, permitindo a sua adaptação aos ambientes de serviços investigados. A partir dos dados obtidos nessa etapa, e com base na revisão da literatura, elaborou-se um questionário estruturado, utilizado na etapa descritiva e conclusiva.

Figura 1 - Modelo teórico sobre antecedentes da lealdade do consumidor, no contexto de recuperação de serviços

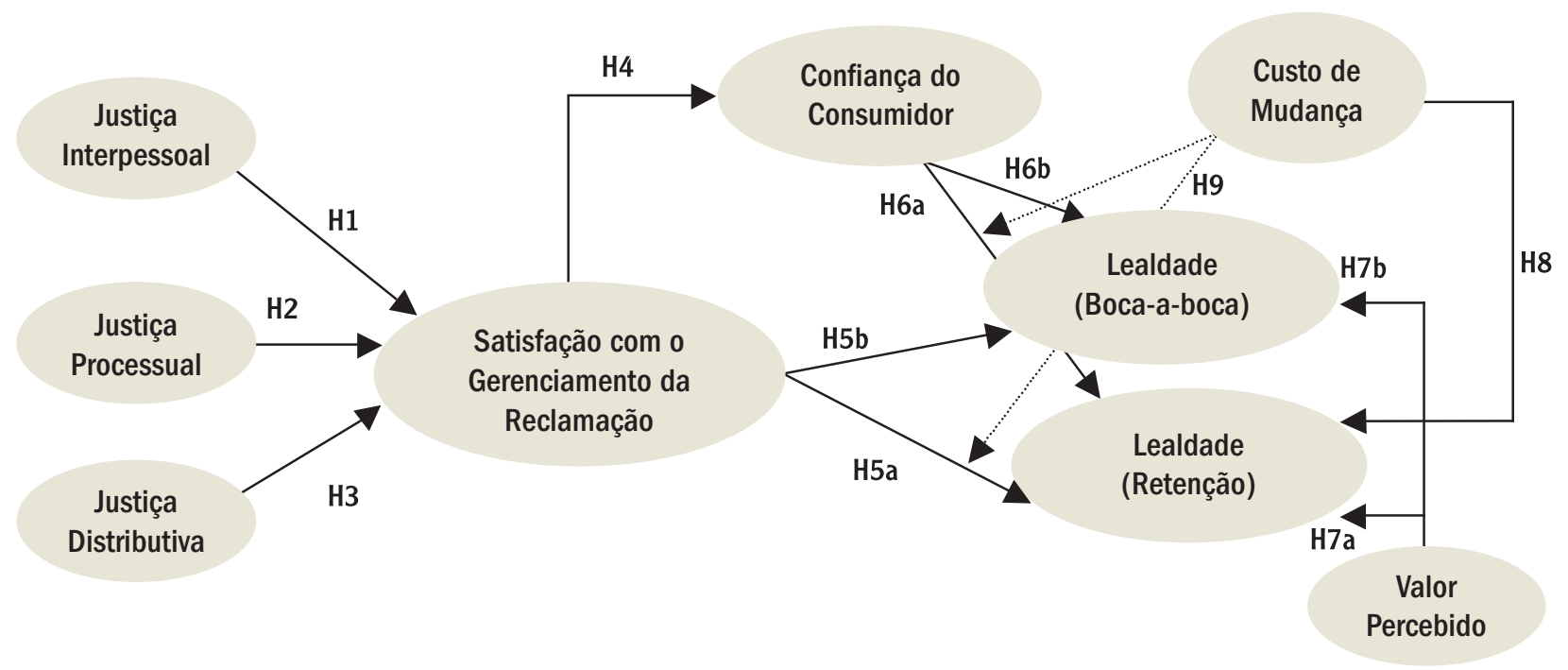




\section{Procedimentos de amostragem e coleta de dados}

Para a etapa quantitativa, a amostra foi de 405 clientes reclamantes: 201 de companhias aéreas e 204 de bancos. Os respondentes foram abordados por entrevistadores no Aeroporto Internacional Salgado Filho, em Porto Alegre. Esse procedimento teve como principal vantagem a possibilidade de se encontrarem clientes de companhias aéreas sem a necessidade de se obter previamente um cadastro deles. Além disso, acreditava-se que haveria uma disposição dos usuários em responder ao questionário nesse local, já que a maioria costumava esperar pelos seus vôos no saguão do aeroporto. Para que o processo fosse o mais aleatório possível, determinaram-se dias e horários variados, incluindo-se horários de vôos noturnos e em finais de semana.

Acreditava-se, também, que pessoas que viajam de avião irremediavelmente utilizariam serviços bancários, pois tendem a ter maior poder aquisitivo do que a maioria da população. Assim, ambas as amostras - reclamantes de bancos e reclamantes de companhias aéreas - foram encontradas no aeroporto. O entrevistador, ao abordar os possíveis entrevistados, questionava se ele havia feito alguma reclamação, nos últimos 12 meses, para uma companhia aérea ou para uma instituição bancária, como pergunta filtro. Os dados foram coletados em março de 2005.

\section{Operacionalização das variáveis}

As questões foram muito semelhantes para as duas indústrias investigadas. Fizeram-se apenas mudanças pontuais, com o intuito de adaptar o questionário a contextos específicos da indústria em questão. Foram utilizadas nesse trabalho as seguintes medidas: justiça interpessoal (6 itens), justiça processual (6 itens), justiça distributiva (4 itens), retiradas de Tax et al. (1998); satisfação com o gerenciamento da reclamação (3 itens), também de Tax et al. (1998); valor percebido (4 itens), confiança do consumidor ( 8 itens; 4 relacionados à confiança nas práticas gerenciais e 4, à confiança nos funcionários), trazidos de Sirdeshmukh et al. (2002); intenção de recompra (3 itens), adaptada de Oliver e Swan (1989), e Zeithaml et al. (1996); comunicação positiva boca-a-boca (3 itens), também de Zeithaml et al. (1996); custos de mudança (4 itens), extraídos de Ping (1993) e Jones et al. (2000). As medidas, originais de estudos norte-americanos, foram traduzidas para a língua portuguesa por meio da técnica de tradução reversa, subseqüentemente submetidas à validação de conteúdo por dois professores doutores em marketing e aplicadas mediante uma escala de 5 pontos (por exemplo, 1 = discordo totalmente; e 5 = concordo totalmente). Previamente à análise, procedeu-se à preparação dos dados. Foram realizadas análises de casos omissos (missing values), extremos (outliers) e de normalidade e homoscedasticidade. Os indicadores de curtose associados às variáveis observáveis sinalizam uma distribuição não normal. Poucos casos, porém, precisaram ser excluídos.

Ressalte-se que, para as dimensões de lealdade, foi coletado apenas o aspecto atitudinal das medidas utilizadas intenções de recompra e recomendação boca-a-boca -, o que se justifica pelo fato de não ser possível obter dados sobre as transações realizadas pelos clientes com o provedor em uma pesquisa do tipo survey. Por outro lado, a coleta apenas da dimensão atitudinal de lealdade implica uma limitação, visto que nem sempre as intenções dos clientes predizem seus comportamentos de compra (dimensão comportamental de lealdade) (DICK; BASU, 1994).

Embora os pesquisadores tenham desenvolvido escalas de custos de mudança mais complexas (ver BURNHAM et al. 2003), o escopo desta pesquisa não permitiu o detalhamento dos diferentes tipos de custo de mudança. Assim, adotou-se uma medida geral de custos de mudança, que buscasse medir a sua intensidade sem detalhar os tipos de custos existentes.

\section{RESULTADOS}

Os resultados deste trabalho se apresentam da seguinte forma. Primeiramente, é realizada uma caracterização geral da amostra. A seguir, examina-se o modelo de medidas por meio da Análise Fatorial Confirmatória (AFC). Somente após o estabelecimento da validade e confiabilidade das medidas utilizadas é que se executa o exame do modelo estrutural. Finalmente, as hipóteses moderadoras são analisadas.

Os programas de computador utilizados foram o SPSS 13.0 e o EQS 5.6, com o método de estimação ERLS (Iteratively Reweighted Generalized Least Squares), seguindo recomendação de Byrne (1994), uma vez que esse método não é baseado no pressuposto de normalidade.

\section{Caracterização da amostra}

As características demográficas das duas amostras foram comparadas por meio da técnica estatística de Análise de Variância. Não tendo sido identificadas diferenças significantes, as amostras foram analisadas conjuntamente.

A média de idade dos respondentes é de 37 anos; e $53 \%$ dos respondentes são homens. Cerca de $45 \%$ dos entrevistados têm renda familiar mensal maior que 4 mil reais; $31,4 \%$, de 2 a 4 mil reais; e 13,1\%, até 2 mil reais. 
A maioria $(68,6 \%)$ tem o terceiro grau completo ou incompleto; e a maior parte das reclamações foi feita pessoalmente $(81,7 \%)$.

Com maior freqüência, os consumidores haviam feito a reclamação nos últimos 6 meses $(50,4 \%)$ anteriores à data da pesquisa, demonstrando que grande parte dos episódios de reclamação era recente. Em relação ao tempo de relacionamento, dentre os reclamantes de bancos, $60 \%$ eram clientes havia mais de três anos; $53 \%$ transacionavam com mais de um banco - sendo que, desses, $62 \%$ consideravam o banco em questão como o principal. Observou-se assim algum grau de relacionamento entre os clientes e os bancos. Entre os que reclamaram para companhias aéreas, $66 \%$ dos entrevistados já haviam utilizado os serviços da empresa mais de três vezes.

Entre os reclamantes de bancos, a média dos custos de mudança foi de 2,72 , enquanto entre os reclamantes de companhias aéreas foi de 2,34. Por meio da Análise de Variância, conclui-se que estas últimas geram menores barreiras de mudanças que os bancos, de acordo com a percepção dos clientes $(F=10,32 ; P<0,01)$. Essa foi a única variável com diferença de média significante entre as duas amostras.

O construto dos custos de mudança obteve a menor média $(2,53)$, enquanto o da confiança nos funcionários conseguiu a maior $(3,56)$, como mostra a Tabela 1 , que apresenta as médias e as correlações entre os construtos investigados.

\section{DISCUSSÃO SOBRE 0 MODELO DE MEDIDAS}

De acordo com autores como Hair et al. (1998) e Churchill (1999), a validade de um modelo de medida é suportada, basicamente, se: (a) o modelo se ajustar aos dados de acordo com índices satisfatórios; (b) as cargas fatoriais dos indicadores nos fatores correspondentes forem altas e significantes; (c) os indicadores de um mesmo construto produzirem índices de confiabilidade superiores a $0,70 \mathrm{e}$ variância extraída acima de 0,50; (d) as correlações entre os indicadores de um mesmo construto produzirem evidência de validade convergente; e (f) a análise das correlações entre os construtos indicar validade discriminante.

Inicialmente, para se testar a invariância do modelo de medidas nos dois tipos de serviço - companhias aéreas e bancos -, esse modelo foi estimado simultaneamente para cada setor, por meio da Modelagem de Equações Estruturais Multigrupos. Seguindo os procedimentos realizados por Sirdeshmukh et al. (2002), inicialmente restringiram-se todos os parâmetros como iguais nos dois grupos, e estimou-se um modelo completamente restrito. Subseqüentemente, com base no teste multiplicador de Lagrange (ver BYRNE, 1994), permitiu-se a variação de parâmetros com indicadores significantes até que não houvesse mais progressos no ajuste do modelo. Nesse caso, não foi identificado nenhum parâmetro cuja variação proporcionasse melhor ajustamento no modelo. Portanto, o modelo de medidas utilizado foi considerado adequa-

Tabela 1 - Média e correlação entre os construtos

\begin{tabular}{|c|c|c|c|c|c|c|c|c|c|c|}
\hline & 1 & 2 & 3 & 4 & 5 & 6 & 7 & 8 & 9 & 10 \\
\hline 1. Valor & 1 & 0,58 & 0,53 & 0,44 & 0,51 & 0,61 & 0,51 & 0,19 & 0,60 & 0,59 \\
\hline 2. Justiça Interpessoal & & 1 & 0,80 & 0,70 & 0,78 & 0,73 & 0,62 & 0,17 & 0,73 & 0,67 \\
\hline 3. Justiça Processual & & & 1 & 0,74 & 0,80 & 0,67 & 0,53 & 0,21 & 0,68 & 0,64 \\
\hline 4. Justiça Distributiva & & & & 1 & 0,88 & 0,66 & 0,52 & 0,14 & 0,64 & 0,54 \\
\hline 5. Satisfação & & & & & 1 & 0,69 & 0,58 & 0,14 & 0,72 & 0,64 \\
\hline 6. Confiança na Empresa & & & & & & 1 & 0,81 & 0,19 & 0,80 & 0,76 \\
\hline 7. Confiança nos Funcionários & & & & & & & 1 & 0,14 & 0,70 & 0,65 \\
\hline 8. Custo de Mudança & & & & & & & & 1 & 0,18 & 0,24 \\
\hline 9. Intenção de Recompra & & & & & & & & & 1 & 0,88 \\
\hline 10. Boca-a-boca & & & & & & & & & & 1 \\
\hline Média & 3,22 & 3,11 & 3,03 & 2,86 & 2,67 & 3,49 & 3,56 & 2,53 & 2,89 & 3,10 \\
\hline Desvio padrão & 1,03 & 1,14 & 1,03 & 1,42 & 1,37 & 1,11 & 1,13 & 1,20 & 1,42 & 1,38 \\
\hline
\end{tabular}


do aos dois tipos de serviços pesquisados. Os índices de ajustamento ao modelo são: $x^{2}=4793.167, \mathrm{gl}=1660, x^{2} /$ $\mathrm{gl}=2,88, \mathrm{NFI}=0,93, \mathrm{NNFI}=0,95, \mathrm{CFI}=0,96$ e RMSEA $=0.06$, todos satisfatórios.

A validade convergente foi suportada pelo fato de que todos os itens apresentaram coeficientes fatoriais altos e significantes nos construtos que se propunham medir (entre 0,59 e 0,96, com valores-t acima de 10,61) Os itens associados a um mesmo construto apresentaram correlações significantes entre si. Foi, portanto, observada a convergência de medidas, isto é, a existência de forte correlação entre as medidas destinadas a mensurar o mesmo construto (CHURCHILL, 1999).

Encontrou-se evidência de validade discriminante, particularmente importante quando os construtos são similares por natureza, nos níveis de correlação existentes entre os construtos. Correlações muito altas indicam falta de validade discriminante, pois os construtos estariam medindo o mesmo fenômeno. Os construtos foram considerados distintos uns dos outros, com a maior correlação entre confiança e lealdade $(0,78)$.

As medidas utilizadas apresentam níveis satisfatórios de confiabilidade e de variância extraída. A confiabilidade ficou entre 0,81 e 0,91 (justiça processual e justiça distributiva, respectivamente), e a variância extraída ficou entre 0,46 e 0,73 (justiça processual e comunicação boca-a-boca, respectivamente). Há, portanto, consistência interna entre os múltiplos indicadores de um construto, mostrando que medem de fato um mesmo fenômeno, além de explicarem substancialmente os respectivos construtos latentes.

\section{DISCUSSÃO SOBRE 0 MODELO ESTRUTURAL}

Após o exame e a validação das medidas utilizadas, o foco deste estudo volta-se para a investigação da estrutura teórica desenvolvida, estabelecendo relações entre os construtos teóricos propostos. A investigação do conjunto de hipóteses será feita, primariamente, por meio dos índices de ajustamento do modelo híbrido e da significância e magnitude dos coeficientes estimados. Além disso, um coeficiente de determinação foi estabelecido para cada equação estrutural, similar ao que é encontrado na regressão múltipla, representando a proporção de variância da variável dependente que é explicada pelas variáveis independentes.

Antes, porém, procedeu-se à análise de equações estruturais multigrupos para se verificar se a união dos dados dos dois tipos de serviços - bancos e companhias aéreas - é apropriada ou se devem ser estimados modelos isolados. A vantagem desse procedimento é o aumento de precisão nos parâmetros estimados (GUJARATI, 1988). O teste multiplicador de Lagrange não indicou nenhum parâmetro cuja variação melhorasse o ajuste do modelo. Portanto, a análise foi conduzida com apenas um banco de dados.

Por ser a avaliação de modelos rivais importante para a discussão do ajuste geral de um modelo empírico, propôsse e testou-se um modelo alternativo, com dois parâmetros adicionais. O primeiro diz respeito à relação entre confiança e valor. O papel de mediação realizado pelo valor percebido tem sido explorado por alguns trabalhos na literatura, como os de Sirdeshmukh et al. (2002), e Brei e Rossi (2005). Segundo os primeiros, sem a criação de valor, a confiança não implica lealdade; o efeito da confiança na lealdade seria condicional ao se incremento no valor percebido.

O segundo parâmetro adicionado contempla a relação causal entre custos de mudança e comunicação bocaa-boca, baseando-se em estudo de Lam et al. (2004), que aponta que os custos de mudança se associam parcialmente a programas de fidelidade que oferecem benefícios. O uso de benefícios pode levar o consumidor a recomendar o fornecedor a outros consumidores por meio da comunicação boca-a-boca positiva, de forma a possibilitar a amigos, parentes e conhecidos que também usufruam de tais benefícios.

Após o ajuste dos modelos original e rival, suas estatísticas foram comparadas. A diferença entre os quiquadrados foi de 12 (2 GL a menos para o modelo rival), não sendo, assim, significante. Além disso, os coeficientes de regressão de ambos os parâmetros acrescentados também não apresentaram significância estatística. Os demais indicadores mantiveram-se inalterados em termos práticos, demonstrando que o modelo alternativo não provê aperfeiçoamento de ajustes quando comparado à estrutura teórica previamente proposta, sendo inclusive menos parcimonioso. Assim, o modelo original foi mantido para exame.

Os resultados da análise do modelo estrutural, tendo como amostra os 405 clientes reclamantes, apresentamse na Tabela 2. O valor do qui-quadrado é significante. Porém, por ser esse teste sensível a desvios de normalidade e a amostras grandes, a análise do valor do qui-quadrado deve ser feita em conjunto com outros critérios de ajustamento (HAIR et al., 1998). Verificando-o sobre os graus de liberdade, produz um valor satisfatório de 2,23, menor do que o máximo recomendado, igual a 5 . Os índices de ajustamento CFI, NFI, NNFI, todos acima de 0,90, são satisfatórios, e o RMSEA de 0,05 é aceitável. 
Os efeitos das percepções de justiça interpessoal, processual e distributiva na satisfação com o gerenciamento da reclamação, estabelecidos nas hipóteses $\mathrm{H}_{1}, \mathrm{H}_{2} \mathrm{e}$ $\mathrm{H}_{3}$, respectivamente, foram suportados pelos resultados obtidos. O tratamento pessoal, o processo de resolução e os resultados tangíveis alcançados por meio da reclamação explicaram uma expressiva proporção (90\%) da variância nos níveis de satisfação final do consumidor. As avaliações sobre os processos e o tratamento pessoal dos empregados no gerenciamento das reclamações influenciaram em menor magnitude a satisfação final, com níveis próximos entre si (coeficientes de regressão de 0,23 e 0,20 , respectivamente). Comparando-se os impactos da justiça distributiva aos impactos das demais na satisfação, percebe-se que os resultados tangíveis obtidos por meio de reclamação tiveram maior efeito na satisfação do consumidor $(0,58)$ do que aspectos interpessoais e processuais, corroborando resultados encontrados por Goodwin e Ross (1992), Smith et al. (1999) e Santos e Rossi (2002).

Os resultados também suportam a hipótese $\mathrm{H}_{4}$, que estabelece a relação entre satisfação com o gerenciamento da reclamação e confiança do consumidor. Observou-se um alto poder explanatório dessa variável em relação à variância da confiança do consumidor, igual a $73 \%$. Isso é consistente com a proposição de que, em situações de conflito, percepções específicas sobre o episódio podem afetar a confiança em relação à outra parte envolvida. Quando o consumidor percebe que a empresa agiu de maneira que ele considera adequada na resolução de sua reclamação, atualiza suas impressões sobre a qualidade da empresa, e, assim, seus sentimentos de confiança são reforçados. O contrário acontece quando o consumidor percebe que a empresa lidou de forma negligente e ineficaz com sua reclamação. A relação entre satisfação e confiança parece ainda fortalecer a lógica de que, em situações de conflito, o consumidor pode perceber se, de fato, a empresa o valoriza e investe em sua manutenção como cliente. Em situações de prejuízo para a empresa em prol da satisfação do consumidor, o relacionamento pode ser colocado à prova.

Os impactos da satisfação pós-reclamação, confiança, valor relacional e do custo de mudança na intenção de recompra do consumidor, propostos em $\mathrm{H}_{5 \mathrm{~A}}, \mathrm{H}_{6 \mathrm{~A}}, \mathrm{H}_{7 \mathrm{~A}} \mathrm{e}$ $\mathrm{H}_{8}$, respectivamente, foram confirmados pelos resultados. No entanto, enquanto a confiança do consumidor apresentou efeito expressivo na intenção de recompra do consumidor (coeficiente de 0,85 ), a relação entre satisfação e intenção de recompra apareceu, de certa forma, menor (coeficiente de 0,39 ). Os efeitos do valor rela- cional $(0,13)$ e, principalmente, do custo de mudança $(0,07)$ na intenção de recompra, embora estatisticamente significantes, foram bem menores do que os efeitos das outras duas variáveis. Estes quatro construtos - satisfação, confiança, valor relacional e custo de mudança explicam $90 \%$ da variância nos níveis de lealdade do consumidor.

O forte efeito da confiança na intenção de recompra indica que, aumentando-se a confiança do consumidor, ele passa a acreditar que a empresa continuará a agir de forma consistente e competente ao longo do tempo, reduzindo, assim, os riscos associados à compra de serviços e sustentando a crença de que ele continuará a obter valor em futuros negócios com tal prestador de serviços. Em outras palavras, quanto mais elevada a confiança do consumidor na empresa e em seus funcionários, maior é a probabilidade de o consumidor realizar futuras trocas com a empresa e de manter um relacionamento a longo prazo.

Em relação à comunicação boca-a-boca, os níveis de satisfação pós-reclamação, confiança e valor percebido pelo consumidor parecem impactá-la, conforme previsto pelas hipóteses $\mathrm{H}_{5 \mathrm{~B}}, \mathrm{H}_{6 \mathrm{~B}}$ e $\mathrm{H}_{7 \mathrm{~B}}$. Novamente, a confiança exerceu a maior influência $(0,90)$, enquanto a satisfação teve efeito mais modesto, $(0,32)$ e o valor percebido, embora significante $(0,11)$, pouco influenciou a predisposição do consumidor em se engajar em comunicação boca-a-boca positiva para a empresa. Expressiva proporção da variância desse construto (97\%) é explicada por esses três antecedentes.

As hipóteses de moderação presentes neste trabalho $\left(\mathrm{H}_{9}\right.$ e $\left.\mathrm{H}_{10}\right)$ destacam possíveis diferenças na intensidade das relações estabelecidas entre satisfação e intenção de recompra, e entre confiança e recompra, por conta dos custos de mudança percebidos pelos clientes. Com custos considerados baixos, a influência da satisfação e da confiança na intenção de recompra seria mais forte do que com custos de mudança mais altos. Para que ela seja testada, foi necessário, portanto, que se segmentasse o banco de dados com 405 respondentes entre o grupo de clientes que percebem os custos de mudança como sendo altos (158 respondentes) e o grupo dos que percebem os custos como baixos (188). Para tanto, os valores da média dos itens que mediram os custos de mudança foram usados para segmentar o banco de dados em três partes iguais, escolhendo-se os grupos $1 / 3$ mais alto e $1 / 3$ mais baixo, respectivamente, para refletir a natureza da moderação, que poderia ser ofuscada pela inclusão de valores intermediários da variável.

Novamente, procedeu-se à análise de equações es- 
truturais multigrupo e, com base no teste multiplicador de Lagrange, observou-se que os parâmetros referentes às hipóteses a serem investigadas, relativamente às relações entre satisfação e recompra, e entre confiança e recompra, não variaram entre os grupos. Isso significa que a relação entre essas variáveis não é moderada pelos custos de mudança percebidos, o que refuta as hipóteses 9 e 10.

Tabela 2 - Coeficientes de regressão estimados às relações teóricas estabelecidas no modelo

\begin{tabular}{|c|c|c|}
\hline RELACIONAMENTOS DO MODELO & COEFICIENTE PADRONIZADO DE REGRESSÃO ab & HIPÓTESES \\
\hline \multicolumn{3}{|l|}{$\begin{array}{l}\text { Variável Dependente: } \\
\text { Satisfação com o Gerenciamento da Reclamação }\end{array}$} \\
\hline Justiça Interpessoal & $\mathbf{0 , 2 0}(2.81)$ & $\mathrm{H}_{1}$ \\
\hline Justiça Processual & $\mathbf{0 , 2 3}(2.70)$ & $\mathrm{H}_{2}$ \\
\hline Justiça Distributiva & $\mathbf{0 , 5 8}(11.84)$ & $\mathrm{H}_{3}$ \\
\hline Variância Explicada & 0,90 & \\
\hline \multicolumn{3}{|l|}{$\begin{array}{l}\text { Variável Dependente: } \\
\text { Confiança do Consumidor }\end{array}$} \\
\hline Satisfação com o Gerenciamento da Reclamação & $\mathbf{0 , 8 6}(16,38)$ & $\mathrm{H}_{4}$ \\
\hline Variância Explicada & 0,73 & \\
\hline \multicolumn{3}{|l|}{$\begin{array}{l}\text { Variável Dependente: } \\
\text { Intenção de Recompra }\end{array}$} \\
\hline Satisfação com o Gerenciamento da Reclamação & $0,39(4,04)$ & $\mathrm{H}_{5 \mathrm{~A}}$ \\
\hline Confiança do Consumidor & $\mathbf{0 , 8 5}(10,95)$ & $\mathrm{H}_{6 \mathrm{~A}}$ \\
\hline Valor Percebido & $0,13(3,46)$ & $\mathrm{H}_{7 \mathrm{~A}}$ \\
\hline Custo de Mudança & $\mathbf{0 , 0 7}(2,83)$ & $\mathrm{H}_{8}$ \\
\hline Variância Explicada & 0,91 & \\
\hline \multicolumn{3}{|l|}{$\begin{array}{l}\text { Variável Dependente: } \\
\text { Boca-a-boca positivo }\end{array}$} \\
\hline Satisfação com o Gerenciamento da Reclamação & $\mathbf{0 , 3 2}(3,48)$ & $\mathrm{H}_{5 \mathrm{~B}}$ \\
\hline Confiança do Consumidor & $\mathbf{0 , 9 0}(12,35)$ & $\mathrm{H}_{6 \mathrm{~B}}$ \\
\hline Valor Percebido & $\mathbf{0 , 1 1}(3,36)$ & $\mathrm{H}_{7 \mathrm{~B}}$ \\
\hline Variância Explicada & 0,97 & \\
\hline \multicolumn{3}{|l|}{ Índices de Ajustamento: } \\
\hline$x^{2}$ (Qui-quadrado) & $1759.754(p<0,001)$ & \\
\hline GL (Graus de Liberdade) & 580 & \\
\hline CFI (Comparative Fit Index) & 0,97 & \\
\hline NFI (Normed Fit Index) & 0,96 & \\
\hline NNFI (NonNormed Fit Index) & 0,97 & \\
\hline RMR (Root Mean Sq. Residual) & 0,32 & \\
\hline RMSEA (Root Mean Sq. Error of Approx.) & 0,06 & \\
\hline
\end{tabular}

a. As estimativas apresentadas são do ERLS (iteratively reweighted generalized least squares) usando EQS.

b. $t$-values entre parênteses. Baseado no teste unicaudal: $t$-values $>1,65=p<0,05$; e $t$-values $>2,33=p<0,01$. Coeficientes significantes em negrito (todos ao nível 0,01). 


\section{CONCLUSÕES}

Neste trabalho, a confiança do consumidor emergiu como o fator mais influente nas intenções de recompra e de recomendação da empresa, seguida pela satisfação com o gerenciamento da reclamação. A influência da confiança pode decorrer do fato de, em ambos os setores estudados - bancos e companhias aéreas -, o risco percebido ser grande; caso ocorram falhas, as conseqüências para o cliente são muito arriscadas. Assim, a confiança no prestador de serviços se torna o principal antecedente da recompra e da comunicação boca-a-boca. Não se deve esquecer, porém, que a satisfação tem também efeito indireto na lealdade por meio de seu forte impacto na confiança do consumidor. Dessa forma, infere-se que o gerenciamento das reclamações possui papel fundamental na construção de relacionamentos duradouros entre empresas e consumidores, sendo assim uma ferramenta estratégica. O gerenciamento inadequado da reclamação geraria o chamado duplo desvio, isto é, a empresa falha duas vezes em atender as necessidades de seu cliente, uma vez quando o problema foi gerado e a segunda quando não responde de forma adequada às reclamações feitas. Com isso, a confiança do consumidor é reduzida e potencialmente destruída, levando o cliente buscar a concorrência.

Embora alguns autores tenham identificado o efeito expressivo dos custos de mudança na intenção dos clientes em permanecer com o mesmo fornecedor, efeito esse de magnitude igual ou maior do que o da satisfação (BURNHAM et al., 2003; LAM et al., 2004; CARUANA, 2003), e também evidenciem a possibilidade de os custos de mudanças terem um papel de moderação na relação entre satisfação e lealdade, este trabalho quantificou um fraco impacto dos custos de mudança na recompra, e nenhum efeito moderador dos custos de mudança nas relações entre confiança e recompra, e entre satisfação e recompra. Uma possível explicação para esses resultados é o fato de que, em relacionamentos em que os clientes se engajam em um processo de reclamação, os custos de mudança são secundários em relação à satisfação pós-reclamação e à confiança como antecedentes das intenções de recompra. Outra possível explicação é o baixo nível dos custos de mudança encontrados relativamente aos dois serviços pesquisados (médias em torno de 2,5, abaixo do nível intermediário). Tal resultado vai contra a crença de que os setores investigados apresentariam custos de mudança significativos. Por fim, uma vez que apenas a dimensão atitudinal de lealdade foi investigada, a análise do impacto dos custos de mudança sobre a dimensão comportamental de lealdade poderia apresentar um impacto maior, pois um cliente pode ter atitudes desfavoráveis frente a um provedor, mas, por existirem custos de mudança, continuar comprando desse mesmo provedor.

\section{Implicações acadêmicas e gerenciais}

Sob a perspectiva acadêmica, o modelo clarifica questões relevantes e ainda pouco exploradas no campo de conhecimento considerado, entre as quais: (1) a recuperação dos serviços como ferramenta de relacionamento, afetando a confiança e a lealdade dos clientes; (2) a importância da confiança como antecedente à lealdade; (3) o impacto de variáveis como valor percebido e custos de mudança na lealdade do consumidor; (4) a aplicabilidade de medidas previamente utilizadas em estudos norte-americanas ao contexto brasileiro; (5) a aplicação do modelo em dois ambientes de serviços diferentes; (6) a confiança como um construto multidimensional - confiança nos funcionários e confiança nas práticas gerenciais da empresa; e (7) a lealdade como um construto bidimensional, englobando intenções de recompra e de recomendação da empresa, podendo cada dimensão ser afetada de maneira diferente pelos construtos apontados como antecedentes, particularmente pela mediação dos custos de mudança, que afetaram apenas as intenções de recompra, oferecendo, assim, validade nomológica para a decomposição da lealdade nessas duas dimensões

Do ponto de vista gerencial, algumas contribuições são apresentadas. Primeiramente, o trabalho trata de processos de reclamação, assunto não priorizado e não pesquisado pelas empresas (SINGH; WILKES, 1996; SANTOS; ROSSI, 2002). Os resultados indicam que os clientes utilizam as respostas a suas reclamações como base para estabelecerem suas atitudes frente à empresa, demonstrando que as reclamações representam, além de uma oportunidade para a empresa rever seus processos e melhorá-los, situações propícias para a solidificação de relacionamentos com os clientes. Ainda sob a perspectiva gerencial, a pesquisa sustenta que a criação de mecanismos que impeçam ou dificultem a saída do cliente não são suficientes para mantê-lo quando a empresa não apresenta desempenho adequado frente a episódios de reclamação; o gerenciamento adequado da reclamação, e a conseqüente confiança gerada entre as partes, é uma forma eficaz para o desenvolvimento e a manutenção de relacionamentos a longo prazo.

\section{Limitações e futuras pesquisas}

As contribuições trazidas por este estudo devem ser ponderadas pelas limitações que o cercam. O trabalho 
utilizou-se de uma abordagem de corte transversal, a partir de uma amostra não probabilística, composta por pessoas que se encontravam no aeroporto no momento da coleta de dados. Embora constitua uma limitação, a abordagem não probabilística viabilizou a investigação do presente trabalho e permitiu a coleta e a análise dos construtos pesquisados. Tal abordagem é utilizada por diversas pesquisas importantes na área de marketing (p. ex., TAX et al., 1998; SIRDESHMUKH et al., 2002; AGUSTIN; SINGH, 2005).

Ainda que tenham sido investigados dois ambientes de serviços considerados importantes para a economia do país por proverem a base e a infra-estrutura necessárias para a realização de negócios em outras áreas, sugere-se, a fim de se testar a estabilidade do modelo construído, a aplicação da pesquisa em outros setores, de preferência que apresentem custos de mudança mais altos, como o de telefonia celular, por exemplo, em que a prestação de serviços é contínua, baseada em contratos, e pouco diferenciada (GASTAL, 2005).

Utilizou-se neste estudo escala semelhante à proposta por Ping (1993), que busca medir, de maneira geral, a intensidade dos custos para o consumidor trocar uma empresa por outra, considerando importantes atributos, como dinheiro, tempo e esforço, que em boa parte expressam o que os consumidores consideram ao avaliar a mudança de fornecedor. Ainda assim, esse construto pode ser insuficiente para captar aspectos referentes a custos de mudança específicos de cada categoria de serviço, como, por exemplo, a não pontuação para o programa de milhagens. Nesse sentido, sugere-se o refinamento da escala de custos de mudança por meio da inclusão de novos itens ou da adaptação dos atuais a diferentes contextos. No contexto da telefonia celular, por exemplo, poderiam ser incluídos os custos de mudança de fornecedor relacionados à mudança de tecnologia e à troca do número do telefone (GASTAL, 2005).

Outra recomendação para futuras pesquisas é a análise do impacto dos tipos de relacionamentos entre consumidores e a empresa, cuja orientação pode ser transacional ou relacional, nos construtos estudados, e pode moderar as relações estabelecidas no modelo, conforme realizado em estudo de Garbarino e Johnson (1999).

\section{REFERÊNCIAS}

ABARCA, F; ALDUNATE, F. O banqueiro da América. Revista Exame, São Paulo, n. 290, p. 28-55, 2 dez. 2004.
AGUSTIN, C.; SINGH, J. Curvilinear effects of consumer loyalty determinants in relational exchanges. Journal of Marketing Research, v. 42, n. 1, p. 96-108, 2005

ARIENTE, M.; GALEANO, R.; GIULIANI, A.; FARAH, O.; PIZZINATTO, N. Marketing de convergência: mudanças no transporte aéreo brasileiro. In: SEMINÁRIO DE GESTÃO DE NEGÓCIOS, 2., 2005, Curitiba, 2005

BERRY, L. Retailers with a future. Marketing Management, v. 5, n. 1, p. 39-46, 1995.

BLODGETT, J. G.; HILL, D. J.; TAX, S. S. The effects of distributive, procedural, and interactional justice on postcomplaint behavior. Journal of Retailing, v. 73, n. 2, p. 185-210, 1997

BOWEN, J. Development of a taxonomy of services to gain strategic marketing insights. Journal of Academy of Marketing Science, v. 18, n. 1, p. $43-49,1990$.

BREI, V. A.; ROSSI, C. A. V. Confiança, valor percebido e lealdade em trocas relacionais de serviço: um estudo com usuários de internet banking no Brasil. Revista de Administração Contemporânea, v. 9, n. 2, p. 145-168, 2005.

BROWN, S. W.; COWLES, D. L.; TUTEN, T. L. Service recovery: its value and limitations as a retail strategy. International Journal of Service Industry Management, v. 7, n. 5, p. 32-46, 1996.

BURNHAM, T. A.; FRELS, J. K.; MAHAJAN, V. Consumer switching costs: a typology antecedents, and consequences. Journal of the Academy of Marketing Science, v. 31, n. 2, p. 109-126, 2003

BYRNE, B. M. Structural Equation Modeling with EQS and EQS/Windows. Thousand Oaks: Sage, 1994.

CARUANA, A. The impact of switching costs on customer loyalty: a study among corporate customers of mobile telephony. Journal of Targeting, Measurement and Analysis for Marketing, v. 12, n. 3, p. 256-268, 2003.

CHEBAT, J. C.; SLUSARCZYK, W. How emotions mediate the effects of perceived justice on loyalty in service recovery: an empirical study. Journal of Business Research, v. 58, n. 5, p. 664-73, 2005.

CHIOU, J.; DROGE, C. Service quality, trust, specific asset investment, and expertise: direct and indirect effects in a satisfaction-loyalty framework. Academy of Marketing Science Journal, v. 34, n. 4, p. 613-28, 2006.

CHURCHILL, G. Marketing Research. Orlando: Fryden Press, 1999

CLEMMER, E. C. The Role of Fairness in Customer Satisfaction with Services Maryland, 1988. 242 f. Tese (Doutorado) - University of Maryland, Maryland, 1988.

DICK, A. S.; BASU, K. Customer loyalty: toward and integrated conceptual framework. Journal of the Academy of Marketing Science, v. 22, n. 2, p. 99-113, 1994. 
DONEY, P. M.; CANNON, J. P. An examination of the nature of trust in buyer-seller relationship. Journal of Marketing, v. 61, n. 2, p.35-52, 1997.

DUBE, L.; MAUTE, M. F. Defensive strategies for managing satisfaction and loyalty in the service industry. Psychology \& Marketing, v. 15, n. 8, p. 775-791, 1998.

DWYER, F. R.; SCHURR, P. H.; OH, S. Developing buyer-seller relationships. Journal of Marketing, v. 51, n. 2, p. 11-28, 1987.

FELDMAN, J. M. The king of the hill evolves. Air Transport World, v. 39, n. 9, p. 36-40, 2002.

FORNELL, C. A national customer satisfaction barometer: the Swedish experience. Journal of Marketing, v. 56, n. 1, p. 6-21, 1992.

GARBARINO, E.; JOHNSON, M. The different roles of satisfaction, trust and commitment for relational and transactional consumers. Journal of Marketing, v. 63, n. 2, p. 70-87, 1999.

GASTAL, F. A Influência da satisfação e dos custos de mudança na lealdade do cliente. 2005. 179 f. Dissertação (Mestrado) - Programa de Pós-Graduação em Administração da Escola de Administração da Universidade Federal do Rio Grande do Sul, 2005.

GILLY, M.C.; GELB, B. Post-purchase consumer processes and the complaining consumer. Journal of Consumer Research, v. 9, n. 3, p. 323-328, 1982.

GOODWIN, C.; ROSS, I. Consumer responses to service failures: influence of procedural and interactional fairness perceptions. Journal of Business Research, v. 25, n. 2, p. 149-163, 1992.

GUJARATI, D. N. Basic Econometrics. New York: McGraw-Hill, 1988.

GWINNER, K. P.; GREMLER, D. D.; BITNER, M. J. Relational benefits in service industries: the customer's perspective. Journal of the Academy of Marketing Science, v. 26, n. 2, p. 101-114, 1998.

HAIR, J.; ANDERSON, R. E.; TATHAM, R.; BLACK, W. Multivariate Data Analysis. New Jersey: Prentice Hall, 1998.

JONES, T. O.; MOTHERSBAUGH, D. L.; BEATTY, S. E. Switching barriers and repurchase intentions in services. Journal of Retailing, v. 76, n. 2, p. 259-274, 2000.

LAM, S. Y.; SHANKAR, V.; MURTHY, M. Costumer value, satisfaction, loyalty, and switching costs: an illustration from a business-to-business service context. Journal of the Academy of Marketing Science, v. 42, n. 3 , p. 293-311, 2004

LEE, J.; LEE, J.; FEICK, L. The impact of switching costs on costumer satisfaction-loyalty link: mobile phone service in France. Journal of Services Marketing, v. 15, n. 1, p. 35-48, 2001.
MARTIN, C. L.; SMART, D. T. Consumer experiences calling toll-free corporate hotlines. The Journal of Business Communication, v. 31, n. 3 , p. 195-212, 1994.

MAXHAM, J. G.; NETEMEYER, R. G. Modeling costumer perceptions of complaint handling over time: the effects of perceived justice of satisfactions and intent. Journal of Retailing, v. 78, n. 4, p. 239-252, 2002.

MORGAN, R. M.; HUNT, S. D. The commitment-trust theory of relationship marketing. Journal of Marketing, v. 58, p. 20-38, July 1994.

OLIVER, R. L. Whence consumer loyalty?. Journal of Marketing, v. 63, p. 33-44, 1999.

OLIVER, R. L.; SWAN, J. Consumer perceptions of interpersonal equity and satisfaction in transactions: a field survey approach. Journal of Marketing, v. 53, n. 2, p. 21-35, 1989.

PING, R. A. The effect of satisfaction and structural constraints on retailing exiting, voice, loyalty opportunism and neglect. Journal of Retailing, v. 69, n. 3, p. 320-352, 1993.

REICHHELD, F. F.; SASSER, W. E. Zero defections: quality comes to services. Harvard Business Review, v. 68, n. 5, p. 105-111, 1990.

RINGBERG, T.; ODEKERKEN, G.; CHRISTENSEN, G. A cultural models approach to service recovery. Journal of Marketing, v. 71, n. 3, p. 194-211, 2007.

SANTOS, C. P.; ROSSI, C. A. V. The impact of complaint handling on consumer's trust and loyalty in the context of relational services exchanges. In: EUROPEAN MARKETING ACADEMY CONFERENCE, 32., 2002. Glasgow. EMAC, 2002

SINGH, J.; WILKES, R. E. When consumer complain: a path analysis of consumer complaint responses estimates. Journal of the Academy of Marketing Science, v. 24, n. 4, p. 350-365, 1996.

SIRDESHMUKH, D.; SINGH, S.; SABOL, B. Consumer trust, value and loyalty in relational exchanges. Journal of Marketing, v. 66, n. 1, p. 1538,2002

SLONGO, L. C; LIBERALI, G. (Org.) Marketing de relacionamento: estudos, cases e proposições de pesquisa. São Paulo: Atlas, 2004.

SMITH, A. K.; BOLTON, R. N.; WAGNER, J. A model of customer satisfaction with service encounter involving failure and recovery. Journal of Marketing Research, v. 36, n. 3, p. 356-372, 1999.

STEPHENS, N.; GWINNER, K. P. Why don't some people complain? a cognitive-emotive process model of consumer complaint behavior. Academy of Marketing Science, v. 26, n. 3, p. 172-189, 1998.

TAX, S. S.; BROWN, S. W.; CHANDRASHEKARAN, M. Customer evaluations of service complaint experiences: implications for relationship marketing. Journal of Marketing, v. 62, n. 2, p. 60-76, 1998.

THIBAUT, J.; KELLEY, H. H. The Social Psychology of Groups. New York: Wiley, 1959. 
THIBAUT, J.; WALKER, L. Procedural Justice: A Psychological Analysis. Hillsdale: Lawrence Erlbaum, 1975.

ZEITHAML, V. A.; BERRY, L. L.; PARASURAMAN, A. The behavioral consequences of service quality. Journal of Marketing, v. 60, n. 2, p. 3146, 1996.
WEBSTER, C.; SUNDARAM, D. S. Service consumption criticality in failure recovery. Journal of Business Research, v. 41, n. 2, p. 153-159, 1998.

WESTBROOK, R. A. Product/consumption-based affective responses and postpurchase processes. Journal of Marketing Research, v. 24, n. 3, p. $258-270,1987$.

Artigo recebido em 09.12.2005. Aprovado em 30.11.2006.

\section{Cristiane Pizzutti dos Santos}

Professora da Universidade Federal do Rio Grande do Sul.

Doutora em Administração de Empresas pela Universidade Federal do Rio Grande do Sul.

Interesses de pesquisas nas áreas de comportamento do consumidor, insatisfação do consumidor e recuperação de serviços.

E-mail: cpsantos@ea.ufrgs.br

Endereço: Rua Washington Luis, 855, sala 454, Centro, Porto Alegre - RS, 90010-460.

\section{Daniel Von der Heyde Fernandes}

Mestrando em Administração de Empresas com ênfase em Marketing pela Universidade Federal do Rio Grande do Sul.

Interesses de pesquisas nas áreas de comportamento do consumidor, insatisfação do consumidor, comportamentos de retaliação e recuperação de serviços.

E-mail: dvon@terra.com.br

Endereço: Rua Washington Luis, 855, sala dos mestrandos, Centro, Porto Alegre - RS, 90010-460. 